\title{
Mycogenic Formation of Silver Nanoparticles by the Azerbaijanese Environmental Isolate Candida macedoniensis BDU-MI44
}

\author{
Ganbarov Khudaverdi Ganbar, Jafarov \\ Mirmusa Mirish, Hajiyeva Fidan Telman, \\ Huseynova Sanam Ismail, \\ Bozkurt Khadija Jalal
}

Faculty of Biology/Department of Microbiology Baku State University

Baku, Azerbaijan

khganbarov1949@bsu.edu.az

\author{
Ramazanov Mohammadali Akhmed, \\ Agamaliyev Zokhrab Adalat, Eyvazova \\ Goncha Malik, \\ Akhmedov Ismat Suleyman
}

Physics faculty/Department of Physical Chemistry of Nanomaterials Baku State University

Baku, Azerbaijan

nanomaterials@bsu.az

\begin{abstract}
Presented work was dedicated to synthesis of silver nanoparticles by yeast strain Candida macedoniensis BDU-MI44, which was procured from the culture collection of the Department of Microbiology. It was founded that this yeast strain is capable of forming silver nanoparticles. First of all its ability to form silver nanoparticles were detected by to stain reaction medium in a dark color and by to give absorption wavelength of $405 \mathrm{~nm}$ in UV-spectrophotometer. In the electron microscope the nanoparticles are observed with a spherical shape and its sizes are $14-23 \mathrm{~nm}$. According to the characteristic X-ray spectrum specified that these nanoparticles are silver.
\end{abstract}

Keywords: silver nanoparticles, yeast, Candida macedoniensis BDU-MI 44, scanning electron microscope, EDS analysis.

\section{INTRODUCTION}

As a result of the intensive development of nanotechnology is approximately $1-100 \mathrm{~nm}$ size nanoparticles have been synthesized and are widely used in various fields of human activity today. As an example of these, can be shown precious metals; gold and silver, non-ferreus heavy metal and metal oxides, semiconductor and organic nanoparticles [1]. These nanoparticles have been widely used in medical diagnosis and treatment, as bearers of drugs, in cosmetics, in production of dyes, in production and packaging of food products, in transportation of products, in oil extraction industry, in agriculture and eventually the protection of the environment More than the synthesis of metal nanoparticles is carried out with the use of physical, chemical and biological methods. Obtaining of nanoparticles by physical and chemical methods is not considered ecofriendly [2]. The majority of the technical processes used in this case can be caused by environmental pollution, which occur with the use of chemical reagents From this point of view, greater attention is paid to the synthesis of nanoparticles through biological objects $[3,4]$. The biological method of nanoparticle synthesis is a relatively simple, cheap, and environmentally friendly method [5].

It is known that many biological systems such as bacteria, fungi, yeast and molds, plants have been used for the convenient biosynthesis of metal nanoparticles. The use of microorganisms for synthesis of nanoparticles is potentially advantageous over plants due to the ease of scaling up the biohazards $[6,7]$. Yeasts are further easy to culture, maintain and due to considerable biomass favor the large scale production of a wide variety of metal nanoparticles including those of silver, gold, iron, cadmium, selenium, platinum and palladium $[8,9,10]$. Metal nanoparticles are characterized by different properties. Silver nanoparticles have recently attracted a lot of interest due to their distinctive properties such as large surface areas, unique physical, chemical and biological properties [11 - 15]. The possibility of synthesis of metal nanoparticles using Candida guilliermondii, C. utilis, C. albicans and C. maltosa was studied in detail [16]. 
The aim of the present work is study the ability of yeast Candida macedoniensis BDU-MI44 synthesize silver nanoparticles.

\section{MATERIALS AND METHODS}

\subsection{Culture and Media}

As an object of research Candida macedoniensis BDU-MI44 used for the synthesis of silver nanoparticles, which was procured from cultures collection of Department of Microbiology. This strain was cultured on malt extract agar slants at $30^{\circ} \mathrm{C}$ for 3 days.

\subsection{Biosynthesis of Silver Nanoparticles}

Candida macedoniensis BDU-MI 44 was inoculated into $250 \mathrm{ml}$ Erlenmeyer flasks, each containing $100 \mathrm{ml}$ of defined medium composed of yeast extract $(10 \mathrm{~g})$, peptone $(20 \mathrm{~g})$, sucrose $(20 \mathrm{~g})$ per 1.000 $\mathrm{ml}$ distilled water at $30^{\circ} \mathrm{C}$ for 48 hours. After cultivation the cultures were centrifuged at $15.000 \mathrm{rpm}$ for $10 \mathrm{~min}$ and $10 \mathrm{~g}$ mycelial biomass was separated from the cultural broth. The settled biomass was washed thrice with $100 \mathrm{ml}$ distilled water. Then $10 \mathrm{~g}$ washed biomass was resuspended in $100 \mathrm{ml}$ distilled water. The cell filtrate and biomass both were challenged with $1 \mathrm{ml}(1 \mathrm{mM}) \mathrm{AgNO}_{3}$ solution. The mixture was incubated in thermostat at $30^{\circ} \mathrm{C}$ for 7 days.

\subsection{Characterization of Silver Nanoparticles}

After 6 hours of incubation the mixture was separated by filtration and presences of silver nanoparticles were analyzed with this sample at filtrate. The bioreduction of silver nitrate into AgNPs was identified by the change of the colour of the cell free filtrate from yellowish to dark brown over a period time. This sample was later subjected to optical measurement, which was carried out by using UV-Visible spectrophotometer ("UV - VIS specord 250 plus", Germany) and scanning the spectra between 300 and $650 \mathrm{~nm}$ at the resalution of $1 \mathrm{~nm}$. The UV-Visible spectra, which represent surface resonance absorption band of Ag-NPs was recorded using UV-Visible spectrophotometer.

A scanning electron microscope (JEOL 7600F, Japan) was used to record the micrograph images of synthesized Ag-NPs. For this purpose, the sample suspension was prepared from cultural broth and was completely evaporated. After preparation, the sample was placed on a film on a support grid and was examined under SEM.

Elemental compotision of synthesized nanoparticles was determined by characteristic X-ray (EDX) spectra.

\section{RESUlTS AND Discussions}

Nanotechnology is the prompt emerging discipline in the field of life science. Biologists are highly interested in synthesizing bionanoparticles using many of the precious metals. A comprehensive study of extracellular biosynthesis of silver nanoparticles ( Ag-NPs) from yeast was carried out in this research work. The isolated pure cultura of Candida macedoniensis BDU-MI44 was inoculated in YPS broth and the obtained cell filtrate was subjected to $1 \mathrm{mM} \mathrm{AgNO}_{3}$. The bioreduction was started within 60 minutes of incubation. Through the researchs it was determined that color of wet biomass and reaction medium darkened during the incubation period. The yellowish colored solution changed into dark brown was clearly observed that the formation of silver nanoparticles. The control flask was incubated in the same condition and there was no colour change appeared (Fig.1). The brown colour of the medium could be due to the excitation of surface plasmon vibration of Ag-NPs. The exact mexchanism of biosynthesis of Ag-NPs is not known. However, is has been hypothesized that silver ions required the NADPH-dependent nitrat reductase enzyme for their reduction, which was secreted by the microorganisms in its extracellular enviroment. The use of this enzyme has previously anaerobic condition. Nitrate reductase is known to shuttle electron from nitrate to the metal group. Thus, these results substantiate the role of nitrate reductase enzyme in biosynthesis of silver nanoparticles.

The synthesized Ag-NPs and its stability were motiored by UV-Vis spectral analysis. It was observed that the mycelial cell filtrate treate with $1 \mathrm{mM} \mathrm{AgNO}_{3}$ showed the plasmon resonance peak at $405 \mathrm{~nm}$. Parallel control experiments containing only wet biomass (without $\mathrm{AgNO}_{3}$ ) did not show any absorption at $405 \mathrm{~nm}$ (Fig.2). This absorption is very specific for the silver nanoparticles. Observation of this peak, assigned to a surface. Plasmon is well documented for various metal nanoparticles with sizes ranging from $2-10 \mathrm{~nm}$. 
SEM provides further insight into the morphology and particle size distribution profile of the silver nanoparticles characterized using SEM. The present data obtained from scanning electron microscope showed distinct shape and size of nanoparticles. The particles from Candida macedoniensis BDUMI44 was spherical in shape in the range of 14-23 nm (Fig.3). This was due to the fact that the particle size obtained was augmented from the hydrated capping agents, particularly proteins and also hydrodynamic effect.

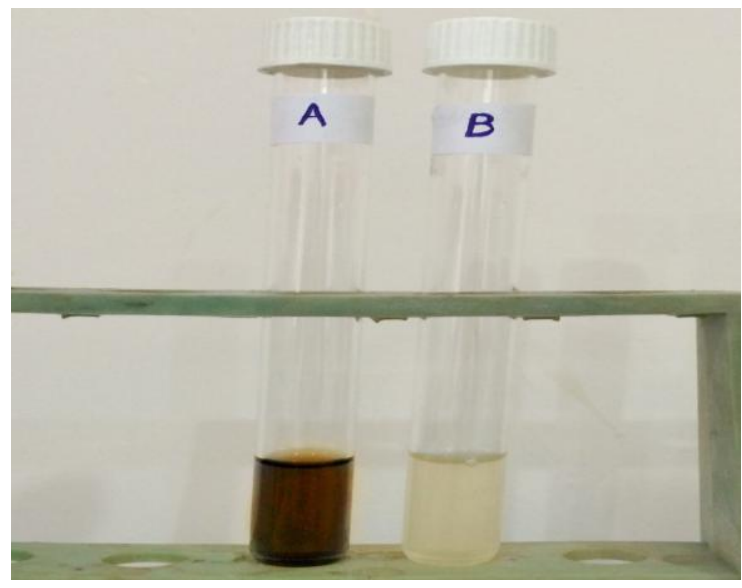

Fig1. The change of color of reaction medium during formation of silver nanoparticles by strain Candida macedoniensis BDU-MI44: a- experimental variant, b-control variant

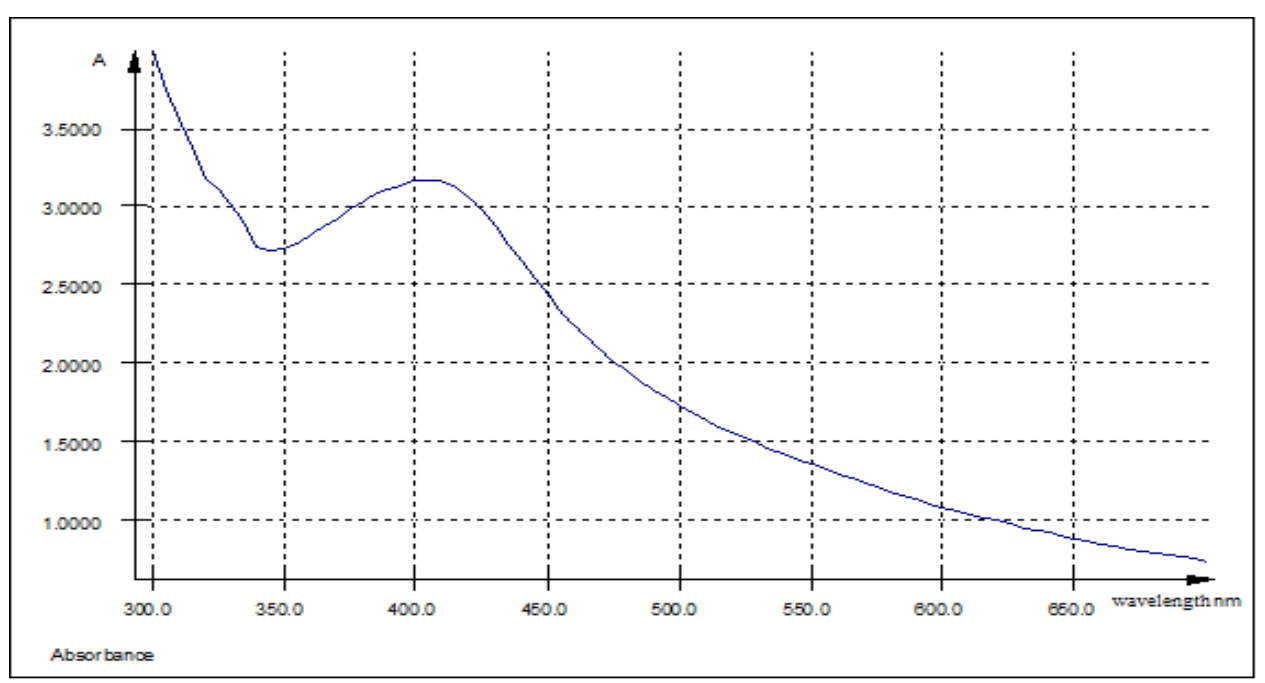

Fig2. UV-visible absorption spectra of silver nanoparticles synthesized by Candida macedoniensis BDU-MI 44 (the peak was found at $405 \mathrm{~nm}$ for 6 hours)

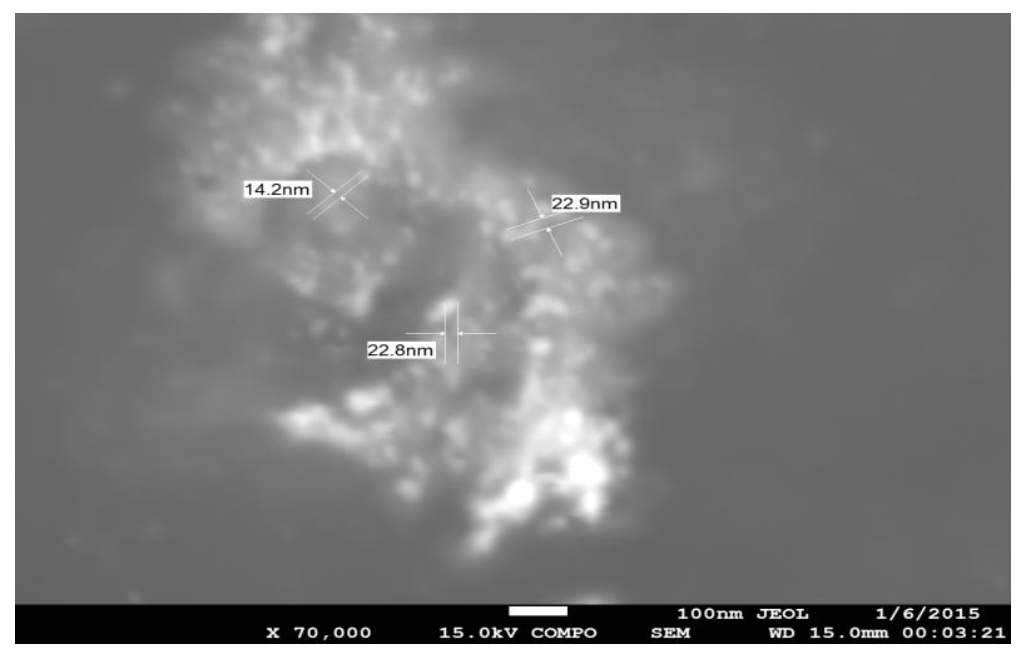

Fig3. Scanning electron microscopy of Ag-NPs synthesized by Candida macedoniensis BDU-MI44 analyzed size and morphology 
The chemical compositions of biosynthesized nanoparticles were determined by X-ray phase analysis that showed strong signal of $\mathrm{Ag}^{\circ}$ with high weight percentage of $\mathrm{Ag}$, as well as $\mathrm{Cl}, \mathrm{O}, \mathrm{Na}, \mathrm{S}$ and $\mathrm{P}$ (Fig.4).

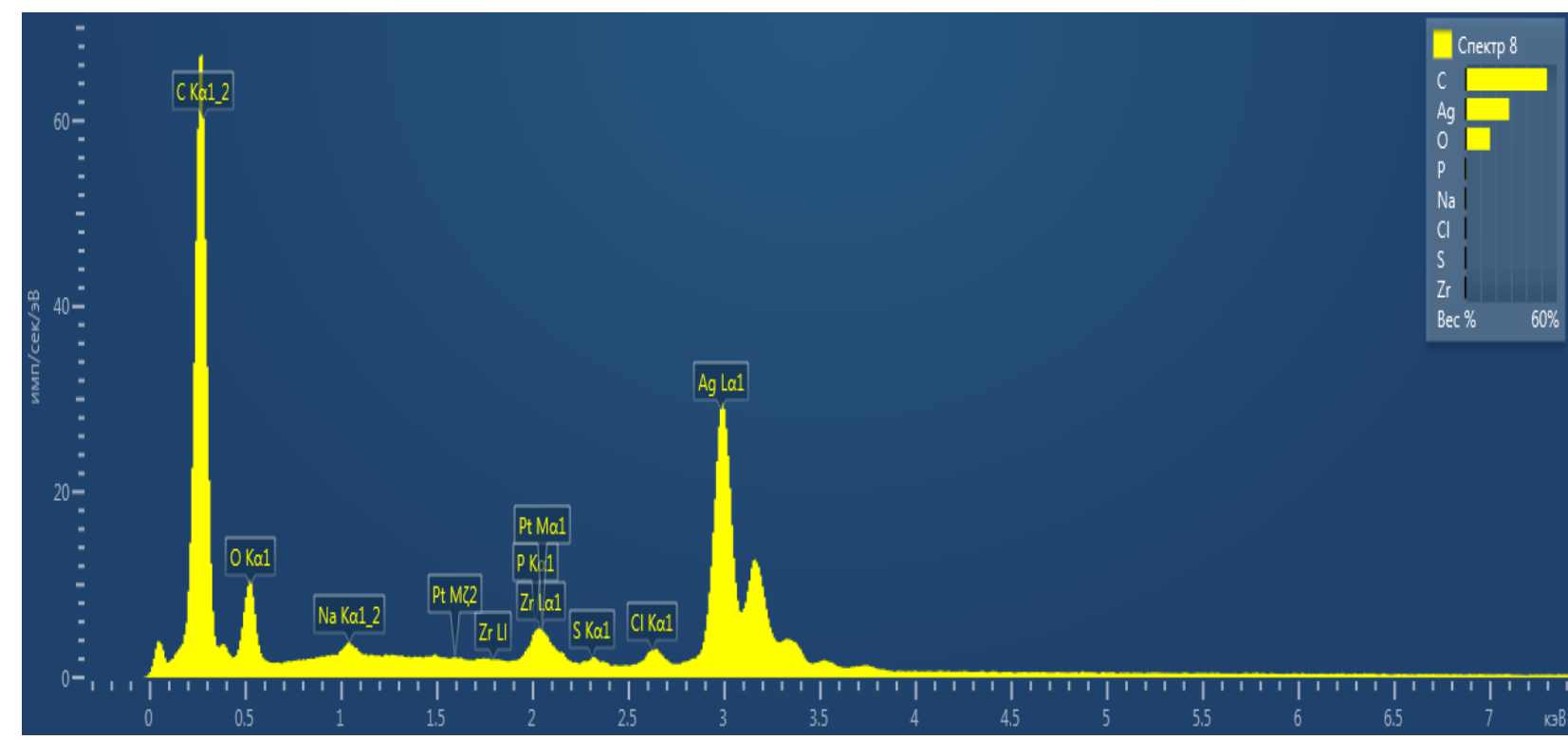

Fig4. The characteristic EDS spectra of silver nanoparticles synthesized by Candida macedoniensis BDU-MI44

\section{Conclusion}

There are many reports that describe methods making use of microbes for the synthesis of nanoparticles as an alternative to cost-ineffective and toxic procedures. The present method determined that the yeast strain Candida macedoniensis BDU-MI 44 capable of producing silver nanoparticles which was procured from the culture collection of the Department of Microbiology. The silver nanoparticles formed by yeast darkened reaction medium, gives an absorption peak at around $405 \mathrm{~nm}$ and it possesses characteristic X-ray spectrum. In the electron microscope the nanoparticles are shown with a spherical shape and its sizes are $14-22 \mathrm{~nm}$. Resulted nanoparticles were smaller in size, uniform, monodispersed in nature, and were synthesized very rapidly.The presented research work explored that due to silver nanoparticles possess excellent physical and chemical properties, they will have great potensials in medical and biological applications. An efficient and simple biosynthesis method to produce silver nanoparticles has been developed by incubating yeast cells with $\mathrm{AgNO}_{3}$. Further studies are required on understanding the cellular and molecular mechanism of AgNPs formation.

\section{REFERENCES}

[1] Ganbarov Kh.G., Ahmadov I.S., Ramazanov M.A., Musayev E.M., Eyvazova G.S., Aghamaliyev Z.A. Silver nanoparticles synthesized by the Azerbaijanian evrironmental isolated Aspergillus niger. Jour. Microbiol. Biotechnol. Food Sciences. 4 (2), 137 - 141 (2014).

[2] Ganbarov Kh.G., Jafarov M.M., Hajiyeva F.T., Bozkurt H.C., Ramazanov M.A., Eyvazova Q.I., Aghamaliyev Z.A., Ahmedov I.S., Abdulhamidova S.M. The study the ability of Candida macedoniensis BDU-MI44 synthesize silver nanoparticles. Jour. Of Qafqaz University. 3 (2), 139-142 (2015).

[3] Ganbarov Kh.G., Ahmadov I.S., Ramazanov M.A., Musayev E.M., Eyvazova G.S., Aghamaliyev Z.A. The concentration effect of the formation of silver nanoparticles by the mold fungus Aspergillus niger BDU A4. Jour. Biotechnol. 185, 28 (2014).

[4] Ghorbani H.R., Safekordi A.A., Atter H., Sorkhabadi S.M. Biological and non-biological methods for silver nanoparticles synthesis. Chem. Biochem. Eng. 25 (3), 317-326 (2011).

[5] Sastry M, Ahmad A, Khan MI and Kumar: Biosynthesis and application of silver and gold nanoparticles. Current Science. 85, 162-170 (2003).

[6] Shailesh R. Waghmare, Mustopa N. Mulla, Suryakant R. Marathe, Kailas D. Sonawane. Ecofriendly production of silver nanoparticles using Candida utilis and its mechanistic action against pathogenic microorganisms. Biotech. 5 (1), 33-38 (2015). 
[7] Zhang S. and Crow S.A. Jr. Toxic Effects of $\mathrm{Ag}(\mathrm{I})$ and $\mathrm{Hg}(\mathrm{II})$ on Candida albicans and C. maltosa: a Flow Cytometric Evaluation. Applied and Environmental Microbiology. 67 (9), 40304035 (2001).

[8] Bharde A., Rautaray D., Bansal V., Ahmad A., Sarkar I., Mohammad Yusuf S., Sanyal M., Sastry M. Extracellular Biosynthesis of Magnetite using Fungi . Biosynthesis of nanoparticles. 2 (1), 135 (2006).

[9] Anal K. Jha, K.Prasad and A.R.Kulkarni. Yeast Mediated Synthesis of Silver Nanoparticles. International Journal of Nanoscience and Nanotechnology. 4 (1), 17-21 (2008).

[10] Egorova E.M, Revina A.A. Synthesis of metallic nanoparticles in reverse micelles in the presence of quercetin. Colloids and Surfaces. A: Physicochemical and Engineering Aspects. 168, 87-96 (2000).

[11] Meenal Kowshik, Shriwas Ashtaputre, Sharmin Kharrazi, W. Vogel, J.Urban, S.K. Kulkarni and K.M. Paknikar. Extracellular synthesis of silver nanoparticles by a silver-tolerant yeast strain MKY3. Nanotechnology. 14 (1), 95-100 (2002).

[12] Narayanan K.B. and Sakthivel N. Biological synthesis of metal nanoparticles by microbes. Advances in Colloid and Interface Science. 156 (1-2), 1-13 (2010).

[13] Sadowski Z. Synthesis of silver nanoparticles using microorganisms. Materials Science Poland. 26, 420-424 (2008).

[14] Sadowski Zygmunt. Biosynthesis and application of silver and gold nanoparticles. Wroclaw University of Technology. 11, 257-266 (2010).

[15] Xiangqian Li, Huizhong Xu, Zhe-Sheng Chen and Guofang Chen. Li, X., Xu, H., Chen, Z.S. and Chen, G. Biosynthesis of nanoparticles by microorganisms and their applications. Jour. Nanomaterials, Article ID 2700974, 1-17 (2011).

[16] Mishra A., Tripathy S.K., Yun S.I. Bio-synthesis of gold and silver nanoparticles from Candida guilliermondii and their antimicrobial effect against pathogenic bacteria. Journal of Nanoscience and Nanotechnology. 1, 243-248 (2011). 